\title{
The Correlated Electronic States of a few Polycyclic Aromatic Hydrocarbons: A Computational study
}

\author{
Geetanjali Giri, Y. Anusooya Pati, and S. Ramasesha*
}

Solid State and Structural Chemistry Unit, Indian Institute of Science, Bangalore 560 012, India

E-mail: ramasesh@sscu.iisc.ernet.in

${ }^{*}$ To whom correspondence should be addressed 
Table 1: Electronic excitation gap and singlet-triplet gaps in $\mathrm{eV}$ for PAH molecules. Numbers in the bracket are experimental gaps obtained from references shown as superscript.

\begin{tabular}{|l|cc|c|}
\hline Molecule & \multicolumn{2}{|c|}{$\begin{array}{c}\text { Optical gap } \\
\text { (Expt gap) }\end{array}$} & $\begin{array}{c}\text { singlet- } \\
\text { triplet gap }\end{array}$ \\
\hline Naphthalene & 6.01 & $(5.63)^{[1]}$ & 2.52 \\
Anthracene & 3.70 & $(3.31)^{[2]}$ & 1.71 \\
Phenanthrene & 4.28 & $(3.60)^{[2]}$ & 2.54 \\
\hline
\end{tabular}

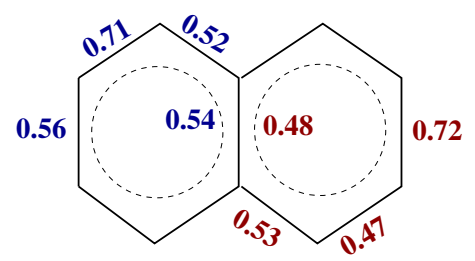

(a)

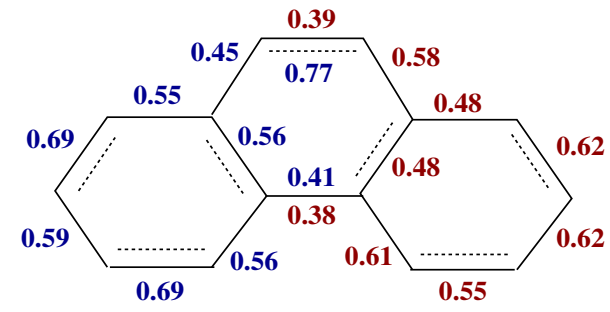

(c)

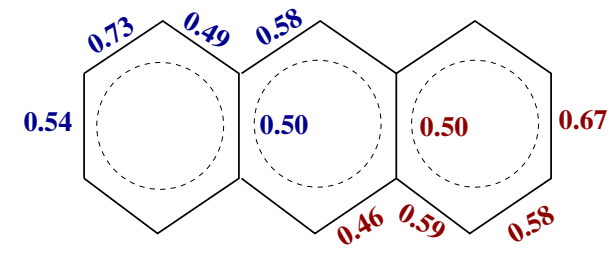

(b)

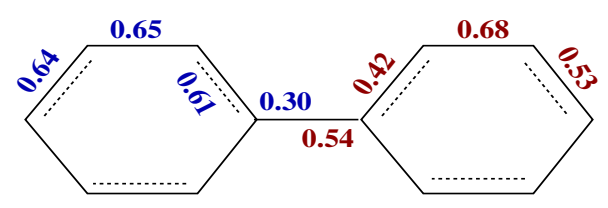

(d)

Figure 1: Bond orders for the lowest singlet (blue in colour) and triplet states for (a) naphthalene, (b) anthracene, (c) phenanthrene and (d) biphenyl (red in colour). 


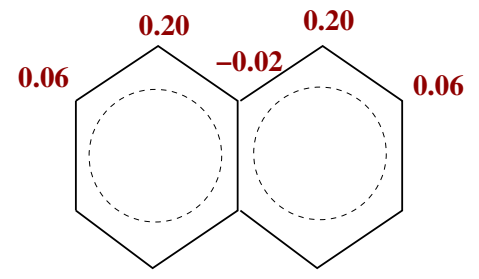

(a)

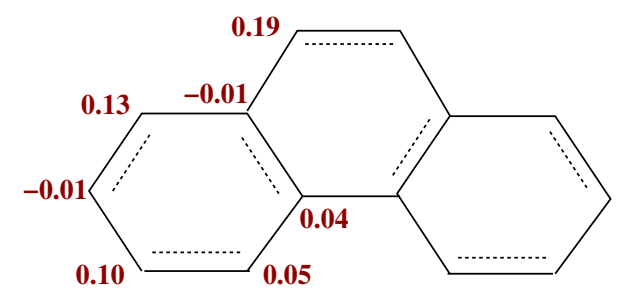

(c)

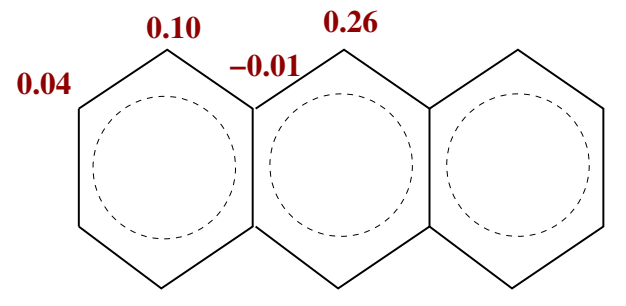

(b)

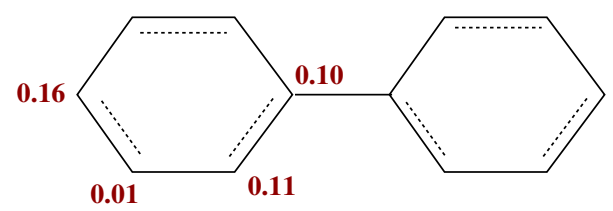

(d)

Figure 2: Spin densities of the lowest triplet states for (a) naphthalene, (b) anthracene, (c) phenanthrene and (d) biphenyl.

\section{References}

1. Birks, J. B.; Christophorou, L. G.; Huebner, R. H.; Excited Electronic States of Benzene and Naphthalene, Nature, 1968, 217, 809-812.

2. Clarence K. Jr. The Longest Wavelength Band in the Electronic Spectra of Polycyclic Aromatic Hydrocarbons for Analytical Use, Appl. Spectr., 1959, 13, 15-25. 